\title{
HONGOS FILAMENTOSOS AISLADOS DESDE EL SUELO Y EL AGUA EN LA PLAYA DE BOA VIAGEM (Recife-Brasil) ${ }^{1}$
}

\author{
Isabel María de Araújo Pinto* \\ M.A.Q. Cavalcanti \\ Departamento de Micología do Centro de Ciencias Biológicas \\ Universidade Federal de Pernambuco \\ José Zanon de Oliveira Passavante \\ Departamento de Oceanografía do Centro de Tecnología \\ Universidade Federal de Pernambuco.
}

Palabras claves: Hongos del suelo y del agua, playa.

Key words: Fungi in soil and water, beach.

\section{RESUMEN}

Se investigó la presencia de hongos filamentosos en disueloy en las aguas de la playa de Boa Viagem, RecifePemambuco, Brasil. Se aisló un total de 10.894 colonias ¿2 hongos.pertenecientes a 49 géneros y 115 especies, mayoritariamente Deuteronycetes. Los géneros Aspergillus $\checkmark$ Penicillium fueron los dominantes en ambos sustrasos:

\section{RESUMO}

Investigou-se a presença de fungos filamentosos no solo e na água da praia de Boa Viagem, PernambucoBrasil. Foi isolado um total de 10.894 colonias de fungos pertencentes a 49 gêneros e 115 espêcies, sendo em sua maioria Deuteromycotina. Aspergillus e Penicillium foram os gêneros dominantes, tanto no solo quanto na Agua.

\section{INTRODUCCION}

La distribución geográfica de los hongos de ambiente marino es esquematizada por Hughes (17) en cinco zonas litorales: ártica, templada, subtropical, zopical y antártica. Escasos e incompletos son los datos sobre la distribución geográfica de esos organismos. La mayor parte de los trabajos están centralizados en Europa y América del Norte y pocos, los tisponible sobre su presencia en los trópicos $(27,29,45)$. En la América del Sur, 43 áreas fueran analizadas en referencia a la existencia de hongos marinos, habiéndose

\section{SUMMARY}

[Filamentous fungi isolated from soil and water in the Boa Viagem beach (Recife-Brasil]

The presence offilamentous fungi was investigated in soil and water of Boa Viagem Beach, Recife-Pernambuco, Brasil. A total of 10.894 c.f.u. representing 49 genera and 115 pecies, most of which identified as Deuteromycetes were isolates. Aspergillus and Penicillium were the dominant genera in both substrates.

detectado 62 especies, de las cuales 20 son citadas para el Brasil (5.20). El reconocimiento de la micota filamentosa del suelo y del agua, en especial de la región marítima abierta, es aún poco explorada. Escasos son los trabajos sobre el tema, destacándose los de Dabrova et al (7), Bergen \& Wagner-Menner (3), Kirk (22), Kishimoto \& Ulken (48) y Udagawa \& Ueda (47). La mayoría de estos se refieren a hongos de ambientes marinos aislados de algas, animales, espuma, vegetales superiores (raices y hojas) yen especial desde anzuelos de lignocelulosa (madera sumergida y flotante $(5,16$, $18,24,27,30,33,36$ y 46 ).

El continente brasileño en su mayor parte, es

1 Parte de la tesis para optar al título de Magister de Isabel de Araújo Pinto.

* Becario del CNPq 
bañado por aguas tropicales, difiriendo en ese punto de otros paises del mundo. Por esto existe gran interés en ampliar los conocimientos sobre los hongos de ambientes marinos del Brasil.

\section{MATERIAL Y METODO}

Se obtuvieron muestras de suelo y del agua de la playa de Boa Viagem, Recife, Pernambuco (Brasil), en el transcurso de un mes, entre el 27 de Enero al 25 de Febrero de 1987. Las colectas fueron realizadas durante la mañana entre las 07:45 y las 11:45 horas, a tres profundidades y en tres estaciones fijas (Fig. 1). Estación 1.- Area poco frecuentada por veraneantes a lo largo de todo el periodo de colecta, con varias dunas cubiertas por algunos vegetales, como: Turnera ulinifolia L., Euphoreria hysophifolia L., Amarantus sp. , Ipomoea pes-capre Sweet, y gramíneas. En la baja mar se visualiza un arrecife continuo, siendo las colectas del suelo y del agua realizadas en el transcurso de todo el periodo entre la playa y el arrecife. Estación 2.- Area con mayor frecuencia de bañistas en el transcurso de todo el período de la colecta. La playa es plana en relación al continente, compuesta de una vegetación deCocos mucifera L. y gramíneas. En baja mar, se visualizan arrecifes no continuos, donde fué posible efectuar las muestras de agua. El suelo cercano a los arrecifes también fué muestreado. Estación 3.- Area carente de vegetación, menos frecuentada por los bañistas durante todo el tiempo de la colecta, con excepción de Sábados y Domingos. En baja mar, el arrecife es continuo y la colecta del suelo se realizó en la parte anterior a éste.

En la región litoral, el suelo fué colectado ( 250 gr.app.) con la ayuda de espátulas estériles, a 5 metros de la demarcación de las aguas en las mareas, ya sea en la superficie $(0-3 \mathrm{~cm})$ o a profundidades de 10 y $20 \mathrm{~cm}$. El material fué depositado en bolsas plásticas estériles.

La colecta del agua fué realizada de acuerdo con la altura de la marea diaria, entre la zona de arrecifes y el litoral, ya sea superficialmente $(0-5 \mathrm{~cm})$ como a profundidades de 0,50 y $1 \mathrm{~m}$.

Se usó una estructura con varillas de madera de $1,70 \mathrm{~m}$. de largo enterrada en dos puntos en el fondo arenoso, con 3 trozos de madera de $20 \mathrm{~cm}$. de largo y $30 \mathrm{~cm}$ de ancho, dispuestos en las 3 profundidades mencionadas. En cada trozo de madera se colocó un tubo de ensayo estéril (en el sentido de la corriente), con tapón de goma y sujeto con tela adhesiva. En el momento de la colecta cuando el tubo de ensayo de la superficie empezó a sumergirse por efecto de la pleamar, los tapones de los 3 tubos fueron retirados y recolocados después de 2 minutos, las muestras de suelo y de agua fueron incubadas a la temperatura ambiente.
Para el aislamiento de los hongos del suelo; de c: muestra se efectuó una suspensión acuosa de $50 \mathrm{~g}$. suelo en $90 \mathrm{ml}$. en agua destilada estéril; usándose : cuotas (previa homogenizacion) de $0,5 \mathrm{ml}$. Para agua se emplearon las mismas alicuotas. Tanto muestras del suelo como del agua fueron sembradas triplicado en Sabouraud-Dextrosa-Agar (34) con . tracto de levadura al 1\%, y cloranfenicol, e incubac a la temperatura ambiente (app. $28^{\circ} \mathrm{C}$ ) entre $7 \mathrm{y}$ : días.

Al iniciarse el desarrollo de las primeras co] nias, éstas fueron repicadas en tubos de ensayo con mismo medio de cultivo. Conforme a los requerimie tos nutricionales y para la obtención de las estructur reproductivas de determinados grupos fúngicos, utilizó además los siguientes medios: PDA, Czape Dox-agar, MA y MA Compuesto.

La identificación se basó en exámenes micro macroscópicos de las colonias y cuando fué necesar microcultivos en láminas (8). Para el reconocimien a nivel del género-especie fueron utilizados los tr bajos de Raper \& Thom (40), Both (4), Ellis (10,11 Gams (12), Raper \& Fennel (39), Domsch et al. (\$ Sutton (43) y Pitt (38).

Durante el período de la colecta del suelo y agu se consideraron algunos parámetros físico-químice tales como: Oxígeno disuelto, altura de las mareas, $T$ pH y salinidad.

\section{RESULTADOS Y DISCUSION}

Mediante las investigaciones realizadas en 54 muestras del suelo y del agua, fueron obtenidas 10.89 . colonias de hongos filamentosos, representados po 49 géneros y 115 especies (41 géneros y 92 especie desde el suelo, y 40 y 93 desde el agua).

La mayor parte de los aislamientos pertenece a li Subdivisión Deuteromycotina, con menor frecuencis Zygomycotina (4\%), Ascomycotina (2\%) y Basidiomy. cotina (1\%). (Tabla 1).

Nuestros resultados coinciden con las obser. vaciones realizadas en suelos marinos de California (7), Hawaii (23) y Florida (3). Garret-Jones (13), considera que casi todos los hongos del ecosistema marino, necesitan del agua para su buena complementación en su ciclo de vida, siendo los Ascomycetes un grupo de buen éxito en las riberas arenosas marinas. Tal situación no ocurre en el presente estudio, pues juntamente Asco y Basidiomycetes fueron los dos grupos que obtuvieron escasos representantes.

De las 3 estaciones, la $N^{\circ} 2$ presenta la mayor variedad y cantidad de hongos del suelo, lo que coincide con su alto índice de bañistas (Tablas 2 y 3 ). Esto 
comprueba lo observado por Bergen \& Wagner-MenDer (3), los cuales citan que todas las playas tienen una enorme variedad en la composición de las especies, - - l las menos frecuentadas por bañistas. En el agua, el - mero más grande de propágulos se detectó en la Estación 3 y la mayor presencia de taxa en la estación 1 (Tablas 2 y 3 ).

La mayor presencia de hongos según las profundidades de las muestras fue, en el suelo a $10 \mathrm{~cm}$, y en el agua en su superficie (Tabla 2 y 3 ). En este aspecto Kirk (22) considera que la superície del oceáno posee una gran cantidad y variedad de hongos que toda la columna del agua.

En la presente investigación observamos que las muestras en las aguas más profundas $(50$ y $100 \mathrm{~cm})$, los registros fueron muy cercanos (Tabla 2), ésto probablemente se deba al hecho de que la mayor parte de las colectas se realizaron en la zona donde rompen las olas.

Entre los géneros de hongos del suelo con más especies (en paréntesis) destacan cuantitativamente los siguientes: Aspergillus (19), Penicillium (17), Phoma (5), Trichoderma (4) Cladosporium y Fusarium (3), Alternaria, Colletotrichum, Dreschslera, Nigrospora, Paecilomyces, Periconia y Pithomyces (2).

En el agua Aspergillus y Penicillium fueron también los dominantes en especies. Subramanian (44), al respecto, cita que Aspergillus y Penicillium son comunes en aguas profundas, pero no en gran número, probablemente debido a que no están presentes las condiciones óptimas de su ciclo de vida. Por otra parte, registramos 79 taxa comunes en el suelo y el agua, 10 que demuestra que probablemente las mareas determinen que los propágulos fúngicos encontrados en las aguas sean depositados en el suelo y viceversa.

Algunas especies de Aspergillus y Penicillium como: A. flavus, A. fumigatus, A. niger, A. sydowi, $A$. tamarii, A. unguis, A. ustus, A. versicolor, A. wentii, $P$. glabrum, P.chermesinum, P. corylophilum, P. implicatum, P.decumbens, $P$. lividum, P.oxalicum, P. paxilii, $P$. steckii, $\boldsymbol{P}$. verruculosum y $\boldsymbol{P}$. vinaceum, fueron encontradas en condiciones hidrológicas muy variables por Bergen \& Wagner-Merner (3), Borut \& Johnson(6), Moustafá \& Al-Musalan(35) y Ristanovia \& Miller(41).

El suelo de la región litoral de la playa de Boa Viagem está constituído en gran parte por arena y cuarzo, pobre en materia orgánica y con $\mathrm{pH}$ alcalino, presenta una cantidad de hongos inferior a la encontrada en suelos ácidos $(21,50)$. Al parecer, el pH no es el único factor que afecta cuantitativamente a los hongos del suelo, pudiendo ser la composición de la materia orgánica el principal factor (37).

En suelos alcalinos algunas de las especies aisla das tales como: Curvularia lunata, C. pallescens, Fusarium oxysporum, $F$. solani, Geotrichum candidum, $M y$ - rothecium roridum, Phoma capitulum, $P$. glomerata, $P$. herbarum, Cladosporium cladosporioides, C. sphaerospermum, Alternaria alternata, A. tenuissima, Paecilomyces variotii, Rhizopus oryzae, Trichoderma viride, Syncephalastrum racemosum, Scopulariopsis brevicaulis y Dreschlera hawaiensis son también referidas por Abdel-Fattah et al. (1), Borut \& Johnson (6), Moustafá \& Al-Musalan (35), Parvar \& Thirumlachar (37). Los hongos patógenos para el hombre, como los citados por Lacaz (34), fueron aislados en los dos substratos, destacándose: Aspergillus fumigatus, A. niger, Geotrichum candidum, Rhizopus oryzae, Scopulariopsis brevicaulis, entre otros. Con excepción de R.oryzae, estos han sido citados en playas de Florida (3), California (7) y Hawaii (23).

Los aislamientos de hongos obtenidos de muestras de la zona intertidal, no significan necesariamente que ellos sean inherentes a esta sub área (7). Es probable que la espuma del mar tenga una función de agente de dispersión de sus esporas (49), originarias de fuentes marinas, estuarinas y terrestres (26). En nuestro, estudio suponemos que algunos de los hongos aislados del suelo y del agua, provenían del estuario de la Playa Barra de Jangada, situada en el Sur, aproximadamente unos $12,2 \mathrm{~km}$ delárea estudiada. Su procedencia puede atribuirse también a procesos de lixiviación del suelo, descargas pluviales, canales domésticos que llevan los detritus fecales a lo largo de la playa, flujo y reflujo de las mareas y actividades de los turistas y bañistas locales. Ulken (48), en sus primeras observaciones, detectó un alto número de hongos en las muestras del fondo de las aguas ecuatoriales de la costa brasileña, la cual es pobre en nutrientes. Algunas de nuestras especies, fueron aisladas anteriormente de habitat terrestres y marinos, aunque se considera a los hongos marinos como especies de ciclos casi exclusivos de este último ambiente (31). Sin embargo aún no existen criterios específicos para distinguir en forma precisa entre ambos $(7,15,42)$. Kohlmeyer \& Kohlmeyer $(31)$, los separa en facultativos y obligados, por el hecho que algunas de las formas marinas crecen mejor en medios preparados con agua de mar u/o agua del mar a diferentes concentraciones y las formas terrestres en agua destilada $(2,7,14,37,42)$. Tricladium angulatum aislado exclusivamente del suelo en la Estación 2, es considerado por Ingold (19) como Hyphomycete acuático.

En ambos sustratos se aislaron cepas no determinadas de Xylariaceae, Aphyllophorales y micelios sin fructificaciones aparentes (hialio y phaeo) (Tabla 1).

Las condiciones hidrologicas presentes durante la época de colecta fueron: para el suelo; marea 0,2-1,9 m; To $24-33^{\circ} \mathrm{C}, \mathrm{pH} 7,85-9,9$; para el agua; marea 0,2 $-1.9 \mathrm{~m}, \mathrm{~T}^{\circ} \cdot 28,25-33^{\circ} \mathrm{C}, \mathrm{DO} 2,92-6,91 \mathrm{ml} / 1, \mathrm{pH} 7,80$ -8.90 y salinidad $30,62-37,12 \%$. 
TABLA 1

Hongos aislados del agua y de la arena de la playa de Boa Viagem, Recife Pernambuco, Brasil.

Absidia cylindrospora Hagen (A-3; S-2,3)

Acremonium bacillisporum (Onions \& BarronW.Gams

(A-1, 3; S-2, 3)

A.rutilum W. Gams (A-2)

Alternaria alternata (Fr.) Keissler (A-1, 2, 3; S-1, 2)

A. tenuissima (Kunze ex Pers.) Witts. (S-2)

Aphyllophorales (A-1, 2, 3; S-1, 2, 3)

Arthrinium phaeospermum (Corda) M. B. Ellis (A-1)

Aspergillus awamori Nakazawa (S-1)

A. bruneo-uniseriatus Singh \& Bakshi $(\mathrm{A}-1,3)$

A. caespitosus Raper \& Thom (A-1; S-2)

A. candidus Link (A-1; S-2)

A. carbonarius (Bain.) Thom (A-1, 2, 3; S-1, 2, 3)

A. carneus Blochwitz ( A-1; S-1)

A. duricaules Raper \& Fennel (S-1,2.3)

A. flavipes (Bain. \& Sant.) Thom \& Church (A-1, 2, 3;

S- $1,2,3$ )

A. flavus Link (A-1, 3; S-1, 2, 3)

A.flavus var. columnaris Raper \& Fennel(A-3; S1,2,3)

A. fumigatus Fres. (A-1, 2, 3; S-1, 2, 3).

A. japonicus Saito (A-2, 3; S-2, 3)

A. nidulans (Eidam) Wint. (A-1; S-3)

A. niger van Tiegh. (A-1, 2; S-1, 2, 3)

A. oryzae (Ahlb.) Cohn (A-2)

A. oryzae (Ahlb.) Cohn var. effusus (Tiraboschi) Ohara (A-1, 3; S-3)

A. puniceus Kwon \& Fennell (A-3; S-2)

A. restrictus $\mathrm{G}$. Smith (A-1)

A. sclerotionum Hubber (A-1, 3)

A. sydowii (Bain, \& Sart.) Thom \& Church (A-1, 2; S-

2)

A. tamarii Kita (A-1, 3; S-1, 2, 3)

A. terreus Thom (A-2; S-1, 2)

A. unguis (Emile-Weil \& Gaudin) Thom \& Raper (S-

1)

A. ustus (Bain.) Thom \& Church (A-1, 2, 3; S-1, 2, 3)

A. versicolor (Vuill.) Tiraboschi (A-3)

A. wentii Wehmer (S-1)

Bartalinia robillardoides Tassi (S-1)

Beauveria alba (Limber) Sacc. (A-1, 2; S-2,3)

Chaetomella raphigera Swift (A-2, 3; S-1, 2, 3)

Chrysonilia sitophila (Montagne) Sacc. (A-1, 2, 3; S-1,

2,3)

Chrysosporium queeslandicum Apinis \& Rees (A-

$1,2,3 ; \mathrm{S}-2)$

Cladosporium cladosporioides (Fres.) de Vries (A-1,

2, 3; S-1, 2, 3)

C. sphaerospermum Penz. (A-1, 2, 3; S-1, 2, 3)

C. tenuissimum Cooke (A-1, 2; S-1,2)

Colletotrichum gloeosporioides (Penz.) Sacc. (S-1, 2,3)
C. lindemuthianum (Sacc. \& Magn.) Br. \& Cav. (t 1,$2 ; \mathrm{S}-1,3$ )

Curvularia lunata (Wakken) Boedijn (A-2,3)

C. pallescens Boedijn (A-1, 2, 3; S-1, 2, 3)

Drechslera australiensis (Bugnicourt) Subram. \&Ja ex M.B.Ellis (S-1)

D. hawaiiensis (Bugnicourt) Subram. \& Jain ex M Ellis (A-1, 2, 3; S-2)

Fusarium lateritium Nees (A-1, 2, 3; S-1, 2, 3)

F. oxysporum Schlecht. (A-1, 2, 3; S-1, 2, 3)

F. solani (Mart.) Sacc. (A-1, 3; S-1, 2, 3)

Geotrichum candidum Link ex Leman (A-2; S-2)

Gliocladium viride Matr. (A-2)

Gliomastix luzulae (Fuckel) Mason ex Hughes (A-:

Glomerella cingulata (Stanem.) Spauld.\& Schrenk।

2)

Hymenopsis trochiloides (Sacc) Sacc. (A-1, 3; S-2)

Lasiodiplodia theobromae (Pat.) Griff. \& Maubl. (S-

Libertella faginea Desm. (A-1,3)

Malbranchea gypsea Sigler \& Carmichael (A-1, 2;

2,3)

Micelio estéril hialino (A-1, 2, 3. S-1, 2, 3)

Micelio estéril dematiáceo (A-1, 2, 3; S-1, 2, 3)

Microsphaeropsis olivacea (Bonord.) Hohm. (A-1, 2,

S-1,2,3)

Mucor microsporus Namyslawski (S-1)

Myrothecium roridum Tode ex Fr. (A-3; S-2,3)

Nigrospora oryzae (Berk. \& Br) Petch (A-1,23; S-1,2,

N. sacchari (Speg.) Mason (A-1, 3; S-2)

Paecilomyces javanicus (Friederichs \& Bally) Borwn

Smith (A-2; S-1, 2, 3)

P. lilacinus (Thom) Samson (A-2)

P. variotii Bain (A-2; S-1, 2,3)

Penicillium brevicompactum Dienckx (A-2)

P. chermesinum Biourge (A-2)

P. chrysogenum Thom (A-3; S-2)

P. citreo-viride Biourge (A-2, 3; S-1, 2, 3)

P. commune Thom (S-3)

P. corylophilum Dierckx $(\mathrm{S}-1,3)$

P. decumbens Thom (A-2)

P. glabrum Westling (A-1, 2, 3; S-1)

P. implicatum Biourge (A-2, 3; S-1, 3)

P. islandicum Sopp. (A-1; S-2)

P. janczewskii Zaleski (A-1,2, 3; S-1, 2, 3)

P. lividum Westling (A-1, 2; S-1)

P. oxalicum Currie \& Thom $8(\mathrm{~A}-1 ; \mathrm{S}-2)$

P. paxilli Bain (A-1), 3; S-1, 2, 3)

P. purpurogenum Stoll (A-1; S-1)

P. restrictum Gilman \& Abbott $(\mathrm{S}-1,2,3$,

P. spinulosum Thom (A-1, 2, 3; S-1, 2, 3) 
P. seckii Zaleski (A-1, 2, 3; S-1, 2, 3)

. variabile Sopp. (A-2)

P. werruculosum Gilman \& Abbot (A-1, 2, 3)

P. vinaceum Gilman \& Abbot $(A-1,2)$

? makmanii Zaleski (A-1, 3; S-1, 2, 3)

?ericonia saraswatipurensis Bilgrami (A-1, 2; S-2)

$P$ tirupatiensis Subram. (A-1, 2, 3; S-1, 2, 3)

Astalotiopsis guepinii (Desm.) Stey. (S-1, 2, 3)

Theeoscytostroma sacchari (Ell. \& Ev.) Sutton (S-2)

Thialophora oxyspora W. Gams (A-1)

Thoma capitulum Pawan Mathur \& Thirum (A-2,3;

$5-3)$

P. exigua Desm. (S-1)

$P$ glomerata (Corda) Wollenw. \& P.glomerata (Corda)

- Illenw. \& Hochapf. (A-2; S-1)

?. herbarum Wested. (A-1, 2, 3; S-1)

P. tropica Schneider \& Boerema (A-1, 2, 3; S-1, 2, 3)

?xhomyces chartarum (Berk. \& Curt.) M.B. Ellis (A-1,

S-1, 2, 3)

Firh. maydicus (Sacc.) M.B. Ellis (S-3)

Peudorobillarda agrostidis (Sprague) Nag Raj, Mogan-

$\therefore$ es \& Kendrick (A-1; S-1)

Ramichloridium subulatum de Hoog (A-2,3; S-1, 2, 3)
Rhizopus oryzae Went. \& Prinsen Geerligs (A-2, 3; S-

2)

Sclerotium sp. (A-2)

Scolecobasidium humicola Barron \& Busch. (A-1).

Scopulariopsis brevicaulis (Sacc.) Bain. (S-1, 2)

Scytalidium lignicola Pesante (A-1; S-1, 2)

Syncephalastrum racemosum Cohn ex Schrot. (A-1,2, 3: S-1, 2,3)

Tetraploa aristata Berk. \& Br. (S-1, 3)

Thielavia terrestris (Apinis) Malloch \& Cain (A-3; S-2)

Thilachlidium brachiatum (Batsch. per Fr.) Petch. (A2; S-1)

Trichoderma aureoviride Rifai (A-1, 2, 3; S-1, 2, 3)

T. harzianum Rifai (A-2, 3; S-1, 2, 3)

T. pseudokoningii Rifai (A-3; S-1, 2,3)

T. viride Pers. ex S.F. Gray (A-1, 2, 3; S-1, 2, 3)

Tricladium angulatum Ingold (S-2)

Verticillium lecanii (Zimm.) Viègas (A-1)

Xylariaceae (A-1, 2, 3; S-1)

A = agua

$\mathrm{S}=$ suelo

Estación = 1, 2, 3.

\section{FIGURA 1}

Ubicación geográfica de las Estaciones de Muestreo

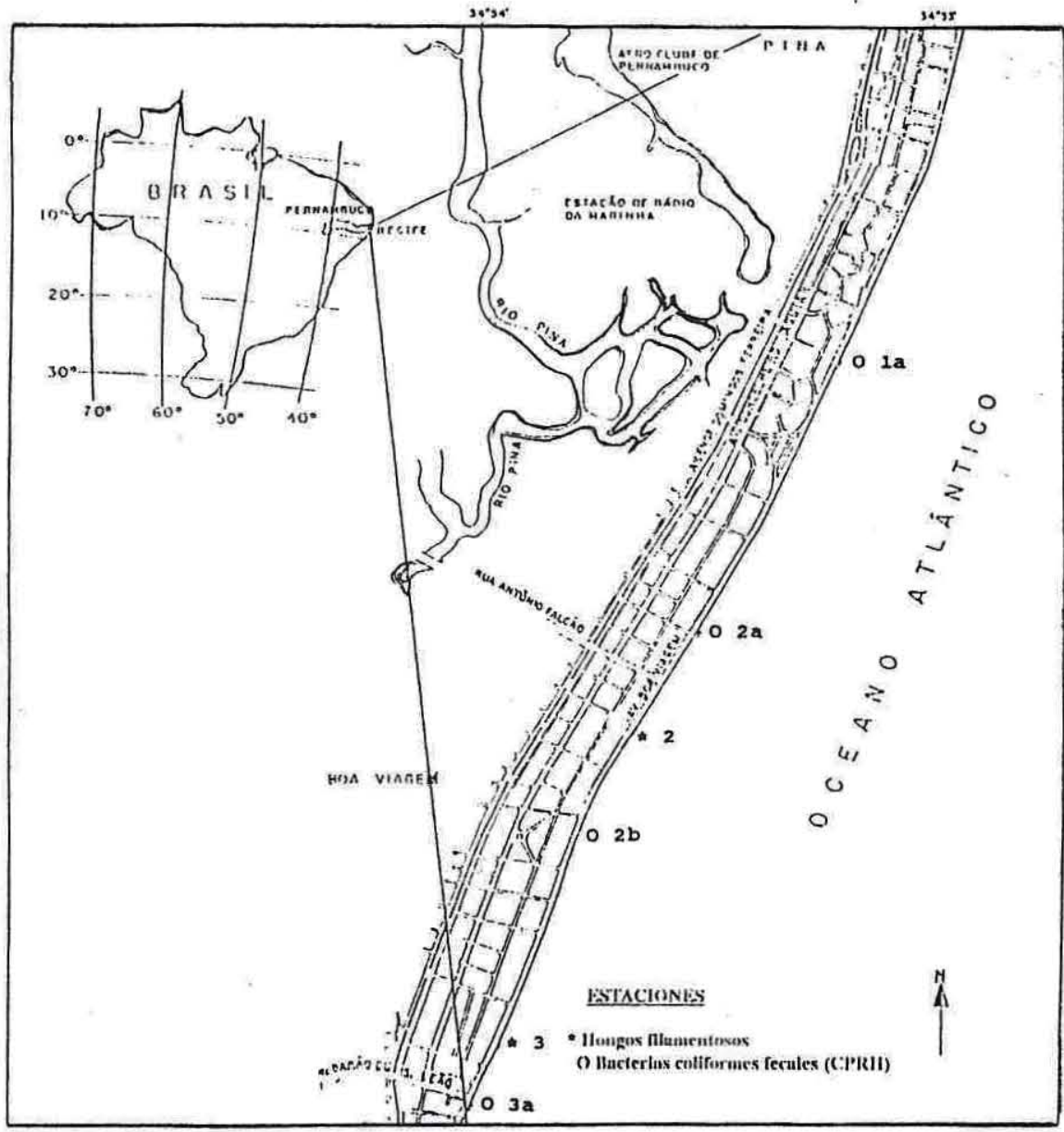


Tabla 2

Número de colonias aisladas desde el agua y desde el suelo (colonias/10 ml) en superficie y a diferentes profundidades.

\begin{tabular}{|c|c|c|c|c|c|c|c|c|}
\hline \multirow[t]{2}{*}{ Estación } & \multicolumn{3}{|c|}{$\begin{array}{c}\text { Substrato } \\
\text { Agua Sup/Prof. (col./10ml) }\end{array}$} & \multirow[t]{2}{*}{ Total } & \multicolumn{3}{|c|}{$\begin{array}{c}\text { Substrato } \\
\text { Suelo Sup./Prof. (col./g) }\end{array}$} & \multirow{2}{*}{ Total } \\
\hline & - & $50 \mathrm{~cm}$ & $100 \mathrm{~cm}$ & & - & $10 \mathrm{~cm}$ & $20 \mathrm{~cm}$ & \\
\hline 1 & 1.011 & 650 & 782 & 2.443 & 140 & 91 & 77 & 308 \\
\hline 2 & 648 & 621 & 896 & 2.165 & 255 & 883 & 707 & 1.840 \\
\hline 3 & 1.229 & 748 & 1.057 & 3.034 & 565 & 234 & 301 & 1.100 \\
\hline Total & 2.888 & 2.023 & 2.735 & 7.646 & 960 & 1.208 & 1.080 & 3.248 \\
\hline
\end{tabular}

Tabla 3

Número de especies aisladas en las Estaciones 1, 2 y 3 en los días de colecta.

\begin{tabular}{|ccc|}
\hline & & Substrato \\
Estación & Agua & Suelo \\
\hline 1 & 66 & 68 \\
2 & 65 & 74 \\
3 & 60 & 56 \\
\hline
\end{tabular}

\section{REFERENCIAS}

1.-Abdel-Fattah, H.M.; Moubasner, A.N.; Abdel-Hafez, S.I. (1977).Studies on mycoflora of salt marshes in Egypt. I. Sugar fungi. Mycopathol. $61: 19-26$.

2.-Barghoorn, E.S. \& Linder, D.H. (1944). Marine fungi their taxonomy and biology. Farlowia, $1: 395-467$.

3.-Bergen,L. \& Wagner-Merner, D.T. (1977). Comparative survey of fungi and potential pathogenic fungi from selected beaches in the Tampa Bay area. Mycologia 69:299-308.

4.-Booth,C. (1971). The genus Fusarium. Commonwealth Mycological Institute. England.

5.-Booth,T. (1979). Strategies for study of fungi in marine and marine influenced ecosystems. Rev. Microbiol. $10: 123-138$.

6.-Borut, S.Y.\& Johnson, T.W. (1962). Some biological observations on fungi in estuarine sediments. Mycologia 54 (2): 181-193.

7.-Dabrowa, N.; Landau, J.W.; Newcorner, V.D.; Plunkett, D.A. (1954). A survey of tide-washed coastal area of southern California for fungi potentially pathogenic to men. Mycopath. Mycol. Appl. 24 : 137-150.

8.-Dalmau, LM. (1929). Remarques sur la technique mycologique. Caracteres macroscopiques des cultures des champignons. Ann. Parasit. Hum. Comp., $7: 536$.

9.-Domsch, K.H, Gams, W. \& Anderson, T-H. (1980). Compendium of soil fungi. London, Academic Press.

10.-Ellis, M.B. (1971). Dematiaceous Hyphomycetes. Commonwealth Mycological Institute. Kew.

11.-_ (1976). More Dematiaceous Hyjphomycetes. Commonwealth Mycological Institute. Kew.

12.-Gams, W. (1971). Cephalosporium artige Schimmelpilze (Hiphomycetes), Gustav Fischer Verlag. Stuttgart.

13.-Gareth Jones, E. B. (1988). Do fungi occur in the sea? The Mycologist. $2: 150-157$.
14. \& Jennings, D.H. (1964). The effect of salinity on th growt h of marine fungi in comparison with non-marine sp cies. Trans. Brit. Mycol. Soc., 47 : 619-625

15.-Grein, A. \& Meyers, S.P.(1958). Growth characteristics and antib. tic production of Ascomycetes isolated from litoral sedime and material suspended in sea water. J. Bact., 76: 457-46:

16.-Haythorn, J.M.; Gareth Jones, E. B. \& Harrison, J.L. (1980). O: servations on marine algicolous fungi, including the Hipt: mycete Signoidea marina sp. nov. Trans. Brit. Mycol. Soc.? 615-623

17.-Hughes, G.C.(1974).Geographical distribution of the higher mari: fungi. Veröff. Inst. Neeresforsch Bremerch. Suppl. 5:419-4

18.-Hyde, K.D. \& Gareth Jones, E.B. (1986). Marine fungi from Seyc elles: II. Lanspora coronata, new genus species from $\mathrm{dr}$. wood. Can. J. Bot. $64: 1581-1585$

19.-Ingoid, C.T. (1942). Aquatic Hyphomycetes of decaying old: leaves. Trans. Brit. Mycol. Soc., $25: 339-417$

20.-Johnson, T.W. Jr. (1967). The stuarine mycoflora. In : Estuarie Washington, D.C., Amer. Assoc. Adv. Sci, pp. 303-305

21.-Jensen, H.L. (1971). The fungus flora of the soil. Soil. Sci., $31: 12$ 158.

22.-Kirk, P.W. Jr. (1983). Direct enumeration of marine arenicolor fungi. Mycol., New York, 75 (4): 670-682.

23.-Kishimoto, R.A \& Baker, G.E. (1969). Pathogenic fungi isolate from beach sands and selected soils of Oahu, Hawaii. Myco New York, 61 (3): 537-548.

24.-Koch, J. (1986). Some lignocolous marine fungi from Thailand, ir cluding two new species. Nord. J. Bot., 6 (4): 497-500.

25.-Koehn, R.D. (1979). A new checklist of Mycelial fungi from marin habitats of Mustang Island, Texas. Southwest Nat., 24 (2): 365-369. 
- Ecological observations on arenicolous manase fungi. Zeitschrift für Allg. Mikrobiologie, Berlin, 6 (2): s. -106 .

2. (1977). Bermuda Marina Fungi. Trans. Brit. Mycol. $503=68$ (2): 207-219.

- (1981). Marine fungi from Eastern Island and notes (1983). Geography of marine fungi. Australian
(19) Jatirnal of Botany, Suppl, 10, 67-76.

$=$ (1985). Marine fungi (Ascomycete) within and on vests of foraminifera, Man. Biol. (Berl.), 90 (1): 147-149.

a. Kohlmeyer, E (1964). Synoptic plates of higher rarine fungi. 2 nd ed. J. Cramer, Weinheim, $64 \mathrm{p}$.

- \& Schatz, S. (1985). Aigialus gen. nov. (Ascomyceve) with two new marine species from mangroves. Trans. Brit. Mycol. Soc, 85 (4): 699-707.

a $\quad$ \& Vitiall, B.P.R. (1986). Lophiastoma mangrovis, a seri marine. Ascomycete from tropics. Mycol., New York, 78 (3): $485-489$.

- C.5.; Porto, E. \& Martins, J. E. C. (1984). Micologia Medica, - ed. Sâo Paulo, Sarvier Editora de Livros Médicos Ittda. 600 .

E Ade A. F. Al.Musallan, A A. (1975). Contribution to the fungal flora of Kwait. Trans, Brit, Mycol. Soc, 65 (3): 547 . 553.

A pres. A.J. \& Fenwick, G.D. (1984). Marine algae and marine fengus, from Open Bay Islands, Westland, New Zeland, N. Z.J. Bot., 22 (3): $425-432$.

Th. V.H. \& Thiremalachar, M. J.(1966). Studies on Halohilic seil fungi from Bombay. Nov. Hedw., Weinheim, $12(3 / 4)$ $497-508$

- 2. (1985). Laboratory guide to common Penichlium spesies. Australia, Commonwealth Scientific and Industrial Research Organization Division of Food Research, 182 p.
39. Raper, X.B \& Tennell, D. I. (1977). The genus Aspergillus. Malabar, Florida, Robert \& Krieger, 68 p.

40.-Raper, K.B. \& Thom, C.A. (1949). Manual of the Penicilia, Baltimore, Williams \& Wilkin, 857 p.

41.-Ristanovi'c, B. \& Miller, C. E. (1969). Salinity tolerances and ecological aspects of some fungi collected from freshwater, estuarine and marine habitats. Mycopathol. Mycol. Appl., 37 (3): $273-280$.

42.-Rilchie, E. (1959). The effect of salinity and temperature on marine and other fungi from various climates. Bull. Torrey Bot. Club, 86:367-373.

43. Sutton, B.C. (1980). The Coelomycetes: Fungi imperfecti with Pic nidia acervulii and stromata. Key, Surrey, England, Commonwealth Mycological Institute, 698 p.

44.-Subramanian, C.V. (1983). Hyphomycetes: Taxonomy and Biology. London, Academic Press. 502 p.

45.-Tan, T.K. (1985). Observations on marine fungi of Singapura and Penang (Malaysia). Trans. Brit. Mycol. Soc, 85 (4): 726 727.

46. Toruga, R (1982). Arenicolous marine fungi from japanese. beaches. Trans. Mycol. Soc. Japan., 7 (4): 423-434.

47.-Udagawa, S. \& Ueda, S. (1985). A new species of Podospora from marine sediment. Mycotaxon, $22(2): 399-406$.

48.-Ulken, A (1966). Söbre a ocorrencia de fungos em amostras do Atlántico Equatorial. Rio de Janeiro, Ministério da Marinha, Instituto de Pesquisas da Marinha. Nota técnica $N^{\circ}$ $28-66,13 \mathrm{p}$.

49. Wagner-Merner, D. T. (1972). Arenicolous fungi from south and central gulf ceoast of Florida. Nov. Hedw., Weinheim, 23 (4): $915-922$.

50. Waksman, S. A (1924). Influence of soil reaction upon the distribution of filamentous fungi in soil. Ecology, 5: 54-59. 